\title{
Microvessel Density (MVD) in Locally Advanced Breast Cancer
}

\author{
Syama Krishnapriya ${ }^{1}$, Basawantrao Malipatil ${ }^{1}$, Suresh Surekha ${ }^{1}$, Shirley \\ Sundersingh ${ }^{2}$, Velusamy Sridevi ${ }^{3}$, Balasubramanian Ananthi ${ }^{4}$, Ganesarajah \\ Selvaluxmy ${ }^{4}$, Trivadi S Ganesan ${ }^{1 *}$
}

\begin{abstract}
Background: The aim of this study was to evaluate microvessel density (MVD) by expression of CD31 and CLEC14A in core biopsies from previously untreated patients with locally advanced breast cancer (LABC) and assess its prognostic significance. Methods: MVD was evaluated in core needle biopsies $(\mathrm{n}=92)$, collected prior to any treatment, from patients who were diagnosed with locally advanced breast cancer (LABC). Immunohistochemistry for expression of CD31 and CLEC14A were performed on these tumours. The median duration of follow-up was 9.3 years. The effect of prognostic factors on disease free survival (DFS) and overall survival (OS) was assessed using a Log rank test and Cox regression model. Results: The clinical factors such as age, clinical nodal stage, stage and pathological nodal status were found to be significant in predicting overall survival by multivariate analysis $(\mathrm{P}<0.05)$. Out of 92,52 tumours had blood vessels expressing CD31, whereas in the remainder, there was no expression. The mean and median MVD of CD31 in 92 tumours was 38 and 5.5 respectively, and it was not a significant factor for predicting disease free survival or overall survival. When we considered the tumours $(n=52)$ which expressed CD31, patients who had very high MVD (>100), had inferior progression free survival and overall survival $(\mathrm{P}=0.5)$. There was no expression of CLEC14A in any of the core needle biopsies whereas it was expressed in specimens from mastectomy from the same patient. Conclusion: This is the first report of MVD in LABC prior to any treatment. The results suggest angiogenesis could be a prognostic factor in LABC.
\end{abstract}

Keywords: CD31- locally advanced breast cancer- Microvessel density- C-type lectin 14A

Asian Pac J Cancer Prev, 20 (5), 1537-1545

\section{Introduction}

Breast cancer is becoming the most frequent type of cancer among women in India. It is the second highest cause of cancer-related mortality among women in the world (Ferlay et al., 2010). The incidence of breast cancer in India nearly ten years ago was 10 per 100,000 women whereas currently it has increased to 23 per 100,000 (Shetty, 2012). The major concern is that despite the technological advances and screening campaign, women present with locally advanced breast cancer (LABC) due to lack of awareness. Among the patients with breast cancer attended our hospital between 1990 and 1999, 69\% of the patients present with LABC (Shanta et al., 2008). The patients who have advanced inoperable large tumours are treated with neoadjuvant concurrent chemoradiation prior to surgery. The disease-free survival (DFS) was $75 \%$ at 5 years in this cohort of patients (Shanta et al., 2008). It is important to understand the pathogenesis of this disease to develop newer approaches to treatment.

Angiogenesis is an essential pre-requisite for the progression of tumours (Folkman, 1971). The research over the past decade has concentrated on identifying a specific marker for tumour blood vessels as they are functionally and morphologically different from their normal counterparts. Under hypoxic conditions inside a tumour, the expression of nearly 2,000 genes changes in the tumour vascular endothelium (Dachs and Chaplin, 1998). During the progression of the tumour, the normal phenotype of the blood vessel changes to an abnormal one by a process called angiogenic switch (Bouck et al., 1996; Hanahan and Folkman, 1996). Targeting tumour endothelial cells requires a surface marker which can specifically identify them (Siemann, 2011). There are several tumour endothelial markers (TEMs) associated with neoangiogenesis (Heath and Bicknell, 2009). The expression is quantified by identifying vessels that express these markers. In breast tumours following mastectomy, it has been shown that high MVD is associated with poor survival (Weidner, 2000; Uzzan et al., 2004). Conventionally, CD31, CD34 and von Willebrand factor (VWF) have been used in evaluating the MVD.

${ }^{1}$ Department of Medical Oncology and Clinical Research, ${ }^{2}$ Department of Pathology, ${ }^{3}$ Department of Surgical Oncology, ${ }^{4}$ Department of Radiation Oncology, Cancer Institute (WIA), 38, Sardar Patel Road, Guindy, Chennai, India. *For Correspondence: tsganesan@gmail.com 
The microvessel counts of CD31 otherwise known as PECAM1 and CD105 has been demonstrated to be an adverse prognostic factor in breast cancer patients (El-Gohary et al., 2007). Recently, other specific TEMs have been identified such as CLEC14A (Mura et al., 2012; Krishna Priya et al., 2017). It has been reported that the expression of CLEC14A was evident only in the tumours whereas it was very low or zero in normal tissues. Although there are many reports evaluating microvessel density as a prognostic factor in breast cancer, these have been largely in operable early breast cancer. In India, as LABC is more common, there have been very few reports on evaluation of microvessel density in this group of patients. Further we wish to evaluate whether it was possible to correlate expression of blood vessels as detected by surface marker expression in a core needle biopsy with other prognostic factors related to outcome. Therefore, we wished to compare the expression of CD31 with that of CLEC14A to assess which would be better in evaluation of microvascular density.

\section{Materials and Methods}

\section{Patients}

Consecutive patients, who presented to the Cancer Institute (WIA) with locally advanced carcinoma of the breast (LABC) from January 2007 to December 2007, were chosen for the study. Locally advanced breast cancer included stage III (A, B, and C). Those patients who were found to have early breast cancer or metastatic disease were not included in the study. The diagnosis was established in all patients by core needle biopsy from the tumor. The paraffin blocks of these patients were obtained from the archive. Each tissue section was checked to ensure that it had at least 50\% malignant cells. The study was approved by Institutional ethics committee. The follow up ranged from 6 months to 10 years with a median follow up of 9.3 years. The patients were followed from January 2008 to December 2017.

\section{Treatment}

Patients with $\mathrm{LABC}$ were treated with the institute protocol of neo-adjuvant treatment (chemoradiation) prior to definitive surgery (modified radical mastectomy or breast conservation surgery). The neoadjuvant chemoradiation protocol included four to six cycles' chemotherapy concurrent with external beam radiation (EBRT) (Shanta et al., 2008).

The chemotherapy which was given q3 weekly included either of the following regimens. These were 5-Fluorouracil, Adriamycin, Cyclophosphamide (FAC), 5-Fluorouracil, Epirubicin and Cyclophosphamide (FEC), Cyclophosphamide, Methotrexate, 5-Fluorouracil (CMF) and Paclitaxel, Epirubicin (TE) according to standard doses (Shanta et al., 2008).

The protocol for concurrent EBRT fields included the whole of the breast, axilla and internal mammary region. All patients received radiation with cobalt beam therapy. Patients with $\mathrm{cN} 1$ axillary nodal disease received radiation to breast and axilla (TD 40Gy, 200cGy/dayx20 fractions) followed by surgery. If there was residual disease in axillary nodes after surgery, those patients received radiation to supraclavicular and internal mammary nodal regions (TD 40Gy). If there were no axillary nodes, radiation to internal mammary nodal regions only was delivered after surgery. In those patients presenting with $\mathrm{cN} 2$ or $\mathrm{cN} 3$ disease, radiation was given to breast, axilla and supraclavicular regions (TD 40Gy) followed by radiation to internal mammary nodal regions (TD 40Gy) after surgery.

EBRT was started with the 1st cycle of chemotherapy in patients who had the cT3N1 disease and among patients with cT4bN0-2 who didn't have a gross breast tissue infection or skin ulceration. Patients with skin ulceration or gross breast tissue infection received 2-3 cycles of chemotherapy initially and radiation was added when a satisfactory reduction in tumor size was documented with a physicians' assessment of tolerance to concurrent radiation. All patients subsequently underwent definitive surgery. Further, these patients received chemotherapy for a total of 6 cycles followed by hormonal therapy i.e., tamoxifen or aromatase inhibitors. The clinicopathological data on all tumours was collected and recorded. Immunohistochemistry

Briefly, formalin fixed paraffin embedded tissues were cut into sections of 4-micron thickness and fixed onto glass slides. The tissue sections were deparaffinized through three changes of xylene followed by rehydration with graded series of alcohol of $100 \%, 90 \%$, and $80 \%$. The tissues were washed with distilled water and further subjected to heat induced epitope retrieval (HIER). The antigen retrieval for CD31 was carried out in a microwave oven by immersing the tissue slides in citrate buffer, $\mathrm{pH}$ 6 for 15 minutes. The tissues were further blocked with hydrogen peroxide and blocking solution (Biogenex) to reduce the non-specific staining. Subsequently, the tissues were incubated with the CD31 antibody (Mouse monoclonal, ready to use, Biogenex) and CLEC14A (Sheep polyclonal, 1:40, R and D systems) for overnight at $4^{\circ} \mathrm{C}$. Next day, the tissue sections were washed with PBS and incubated with anti-mouse HRP antibody (ready to use, Biogenex) for 1 hour. The tissues were incubated with DAB for 15-30 minutes for the development of the brown colour. Further, the tissues were counterstained with hematoxylin and mounted in DPX. The slides were observed under microscope and images were captured (Nikon E200). For CLEC14A, the tissues were subjected to HIER in a water bath (citrate buffer, pH6) for 30 minutes by giving an interval of 5 minutes after the first 15 minutes (Krishna Priya et al., 2017).

\section{Evaluation of microvessel density}

The tissues were small and hot spots could not be identified and the Chalkley method was not used to determine the vascular count. Hence, the total number of stained vessels was counted in each tumour by two independent individuals and the average of both readings was considered for further analysis (Fox and Harris, 2004).

\section{Statistical analysis}

The prognostic factors and their relation to the clinical outcome were evaluated by Cox proportional model 
and the survival curves were plotted by the method of Kaplan-Meier (Kaplan and Meier, 1958; Cox and Oakes, 1984). Overall survival (OS) was defined as the period from diagnosis until date of last follow-up or date of death from any cause. Disease-free survival (DFS) was defined as the period from the date of diagnosis to the date of relapse or death. Pathological complete response is defined as complete absence of tumour in tissues and nodes removed during surgery following radiotherapy or chemotherapy. Univariate and multivariate analysis were performed to evaluate all the factors. In multivariate analysis, since clinical and pathological nodal status is dependent, we have split them into two separate models for analysis which included age and pathological complete responses. All statistical analysis was performed using SPSS version 20. A P value less than 0.05 was considered to be significant.

\section{Results}

\section{Patients}

During January 2007 to December 2007, 237 patients with breast cancer were diagnosed to have LABC. In this study, we have included 135 patients who have received neoadjuvant chemoradiation. The remaining 102 patients were not included due to treatment abandonment, availability of only fine needle aspirate cytology, non-availability of tissues because surgery was done outside and inadequate follow-up. These 135 patients have been included in the study as they all had undergone upfront core needle biopsy followed by neoadjuvant chemoradiation and surgery and had adequate follow-up.

The median age was 47 (range 22-70). There were 98 patients with clinical stage IIIA and 37 were with clinical stage IIIB (Table 1). Patients having ER-positive and negative tumours were 75 (55.5\%) and 58 (42.2\%)

(a)

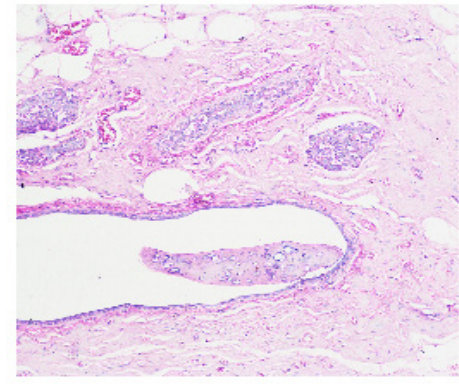

(c)

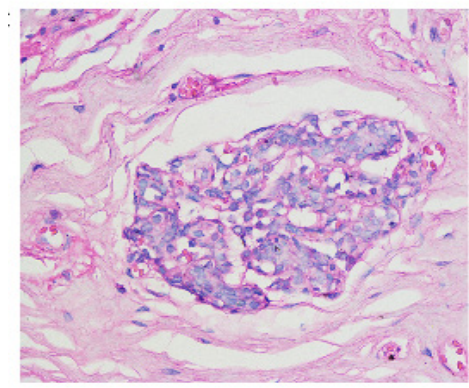

respectively. ER status was not available in 2 patients. Similarly, there were $65(50 \%)$ patients with PR positive tumours and $65(50 \%)$ with PR-negative (PR status for 5 patients was not available). Evaluation of the c-erb2 receptor was not routinely performed on tumours during 2007 and therefore not available. Two patients had grade 1 tumors, 60 were grade 2 tumors and 66 were grade 3 tumors. For 7 patients, the information on the grade of the tumor was not available. For statistical analysis grade, 1 and 2 tumors were grouped as low grade and grade 3 as high grade.

\section{Treatment}

Eighty-nine patients (66\%) received FAC, 22 (16.2\%) FEC-60, 3 (2.2\%) Paclitaxel + Epirubicin and 21 patients (15.5\%) CMF chemotherapy. Concurrent radiation was started with the first cycle of chemotherapy for 119 $(88 \%)$ patients and the remaining 16 patients received radiation in subsequent cycles. Among the 135 patients, $125(92.5 \%)$ patients underwent an Auchincloss type of modified radical mastectomy, 8 patients underwent Patey's mastectomy and one patient underwent segmental mastectomy (breast conservation surgery). One patient progressed on chemoradiation and hence surgery on the breast was not performed and this patient subsequently died of disease. The median number of nodes removed during modified radical mastectomy was 11 (range 3 -25). The median number of nodes positive among those who did not attain pathological complete response (pCR) was 2 (range 1 - 11). Out of 134 patients, $47(35 \%)$ had a pCR on examination of their tumor and nodes at surgery after neoadjuvant chemoradiation.

\section{Survival analysis}

Out of 136, Thirty six patients had relapsed, of which 31 have died. The five-year DFS and OS were $72.5 \%$ and

(b)

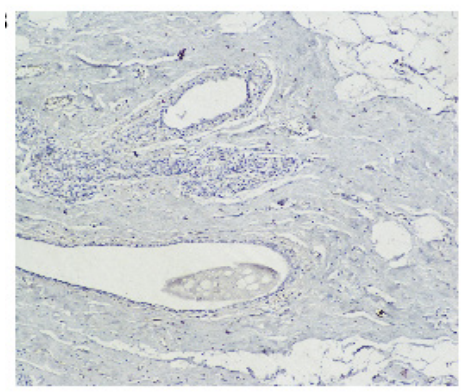

(d)

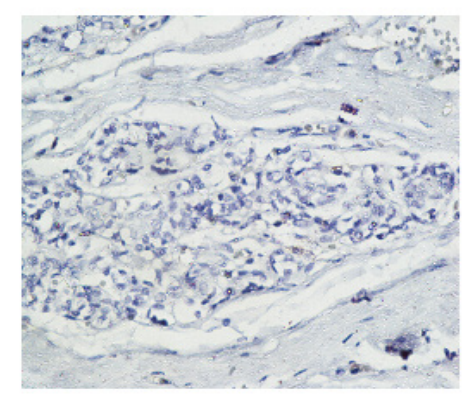

Figure 1. Expression of CD31 in Normal Breast Tissue. Hematoxylin and Eosin stain and IHC of CD31 under magnification 10x ( $\mathrm{a}$ and $\mathrm{b})$, and 40x (c and d) respectively. There was no expression of CD31 in the normal tissue. 
Table 1. Clinicopathological Characteristics of the Patients

\begin{tabular}{|c|c|}
\hline Characteristic & $\mathrm{N}(\%)$ of 135 \\
\hline \multicolumn{2}{|l|}{ Age } \\
\hline $21-30$ years & $5(3)$ \\
\hline $31-40$ years & $30(22.2)$ \\
\hline $41-50$ years & $59(43.7)$ \\
\hline $51-60$ years & $27(20)$ \\
\hline $61-70$ years & $14(10.3)$ \\
\hline \multicolumn{2}{|l|}{ AJCC Stage } \\
\hline IIIA & $98(72)$ \\
\hline IIIB & $37(28)$ \\
\hline IIIC & 0 \\
\hline \multicolumn{2}{|l|}{ T stage } \\
\hline $\mathrm{T} 2$ & $9(6.6 \%)$ \\
\hline $\mathrm{T} 3$ & $89(66 \%)$ \\
\hline $\mathrm{T} 4$ & $37(27 \%)$ \\
\hline \multicolumn{2}{|l|}{$\mathrm{N}$ stage } \\
\hline $\mathrm{N} 1$ & $96(71 \%)$ \\
\hline N2 & $39(29 \%)$ \\
\hline \multicolumn{2}{|l|}{ Grade } \\
\hline 1 & $2(1.5 \%)$ \\
\hline 2 & $60(44.5 \%)$ \\
\hline 3 & $66(49 \%)$ \\
\hline Not evaluable & $7(5 \%)$ \\
\hline \multicolumn{2}{|l|}{ Histological subtypes } \\
\hline Infiltrating ductal carcinoma & $126(93.3)$ \\
\hline Medullary carcinoma & $3(2.2)$ \\
\hline Mucinous carcinoma & $3(2.2)$ \\
\hline Others & $3(2.2)$ \\
\hline \multicolumn{2}{|l|}{ Hormonal status } \\
\hline ER positive & $75(55.5 \%)$ \\
\hline ER negative & $57(42.2 \%)$ \\
\hline PR positive & $65(50 \%)$ \\
\hline PR negative & $65(50 \%)$ \\
\hline Hormonal status not available & $3(2.2 \%)$ \\
\hline \multicolumn{2}{|l|}{ Treatment } \\
\hline Chemo-radiation & $119(88.2 \%)$ \\
\hline Chemotherapy followed by radiation & $16(11.8 \%)$ \\
\hline \multicolumn{2}{|l|}{ Type of chemotherapy } \\
\hline FAC & $89(66 \%)$ \\
\hline FEC-60 & $22(16.2 \%)$ \\
\hline $\mathrm{CMF}$ & $21(15.5 \%)$ \\
\hline Paclitaxel + Epirubicin & $3(2.2 \%)$ \\
\hline \multicolumn{2}{|l|}{ Type of surgery } \\
\hline Modified radical mastectomy & $125(92.5 \%)$ \\
\hline Patey's mastectomy & $8(5.9 \%)$ \\
\hline Segmental mastectomy & $1(0.7 \%)$ \\
\hline Not done & $1(0.7 \%)$ \\
\hline
\end{tabular}

$75 \%$ respectively.

\section{Univariate analysis}

The clinical factors were evaluated by Cox proportional model to understand their influence on the outcome (Table 2). Among these factors, clinical node stage and pathological nodal status $(\mathrm{pN})$ were found to have a significant impact on DFS and OS. The achievement of Pathological complete response was not a significant factor probably due to sample size. On comparison between pathological node negative and positive group, there was a significant survival benefit in both DFS (HR: 2.79; $95 \% \mathrm{CI}, 1.44$ to $5.43 ; \mathrm{P}=0.002$ ) and OS (HR: $3.48 ; 95 \% \mathrm{CI}, 1.75$ to $6.91 ; \mathrm{P}=<0.001)$. Overall, 99 out of 134 patients had a complete pathological absence of tumour in axillary lymph nodes. Seventy-eight out of 96 patients with cN1 was ypN0 and 21 out of $38 \mathrm{cN} 2$ disease patients had pathologically sterile nodes post neoadjuvant chemoradiation. In $\mathrm{cN} 1,20$ out of 96 patients $(20 \%)$ relapsed and with $\mathrm{cN} 2$ disease $16(41 \%)$ of 39 patients relapsed (HR: 2.43; 95\% CI, 1.26 to 4.69 ; $\mathrm{P}=0.008$ ). Nineteen of the $\mathrm{cN} 1$ relapsed patients died and 15 of $\mathrm{cN} 2$ relapsed patients died during the study period (HR: 2.3; $95 \%$ CI, 1.18 to $4.59 ; \mathrm{P}=0.014)$. Similarly, the univariate analysis of the subset of 92 patients, whose tumours were analysed for MVD, showed that clinical node stage and pathological nodal status $(\mathrm{pN})$ were significant prognostic factors (data not shown).

\section{Multivariate analysis}

The factors which were significantly correlated with DFS and OS were considered in the multivariate analysis. Clinical and pathological nodal stages and age were the adverse factors in univariate analysis. Since the clinical and pathological nodal stages were dependent variables, we have performed the multivariate analysis by considering two models. The age, clinical tumour size, and the pathological complete response had an influence on the clinical outcomes and hence were taken as independent variables for multivariate analysis. The pathological nodal stage emerged as the strongest variable for predicting overall survival (HR: $3.15 ; 95 \% \mathrm{CI}, 1.35$ to 7.45; $\mathrm{P}=0.009$ ). The clinical nodal stage (HR: $2.33 ; 95 \%$ CI, 1.16 to $4.65 ; \mathrm{P}=0.017$ ) and age (HR: $0.28 ; 95 \% \mathrm{CI}$, 0.10 to $0.82 ; \mathrm{P}=0.02$ ) were also found to be significant predictors of overall survival. The clinical nodal stage significantly correlated with disease-free survival (HR: 2.44; 95\% CI, 1.24 to $4.79 ; \mathrm{P}=0.009$ ). The pathological nodal stage was also a significant predictor of disease-free survival (HR: $2.67 ; 95 \% \mathrm{CI}, 1.20$ to $5.89 ; \mathrm{P}=0.015$ ). Age was not statistically significant for disease-free survival unlike overall survival (Table 3A and 3B).

\section{Evaluation of MVD by CD31 and its prognostic} significance

Out of 135 patients, 92 paraffin blocks of core needle biopsies could be retrieved with satisfactory tumour tissue from the tumour bank. There was no expression of CD31 in the normal breast tissue (Figure 1). CD31+ blood vessels were observed in 52/92 tumour samples. The tumours that were negative for expression of CD31 
DOI:10.31557/APJCP.2019.20.5.1537

MVD and Locally Advanced Breast Cancer

Table 2. Univariate Analysis of Clinical Factors

\begin{tabular}{|c|c|c|c|c|c|c|c|c|}
\hline \multirow[t]{2}{*}{ Variable } & & \multirow[t]{2}{*}{ Number } & \multicolumn{3}{|c|}{ Disease Free Survival } & \multicolumn{3}{|c|}{ Overall survival } \\
\hline & & & Hazard Ratio & $95 \% \mathrm{CI}$ & P Value & Hazard Ratio & $95 \% \mathrm{CI}$ & $\mathrm{P}$ Value \\
\hline Age (Continuous) & & 135 & 0.965 & $0.934-0.998$ & 0.036 & 0.955 & $0.922-0.989$ & 0.009 \\
\hline \multirow[t]{2}{*}{ Age (Categorical) } & $<50$ years & 94 & 1 & $0.218-1.136$ & 0.097 & 1 & $0.099-0.802$ & 0.018 \\
\hline & $>50$ years & 41 & 0.497 & & & 0.282 & & \\
\hline \multirow[t]{2}{*}{ Clinical Node Stage } & 1 & 96 & 1 & $1.255-4.689$ & 0.008 & 1 & $1.184-4.592$ & 0.014 \\
\hline & 2 & 39 & 2.426 & & & 2.331 & & \\
\hline \multirow[t]{2}{*}{ Clinical stage } & IIIA & 98 & 1 & $0.645-2.670$ & 0.453 & 1 & $1.102-4.327$ & 0.025 \\
\hline & IIIB & 37 & 1.313 & & & 2.184 & & \\
\hline \multirow[t]{2}{*}{ Grade } & Low & 62 & 1 & $0.546-2.057$ & 0.864 & 1 & $0.668-2.660$ & 0.414 \\
\hline & High & 66 & 1.06 & & & 1.333 & & \\
\hline \multirow[t]{2}{*}{ ER } & Positive & 75 & 1 & $0.646-2.393$ & 0.514 & 1 & $0.508-2.02$ & 0.972 \\
\hline & Negative & 58 & 1.244 & & & 1.013 & & \\
\hline \multirow[t]{2}{*}{ PR } & Positive & 65 & 1 & $0.801-3.015$ & 0.193 & 1 & $0.749-2.980$ & 0.255 \\
\hline & Negative & 65 & 1.554 & & & 1.493 & & \\
\hline Neoadjuvant & Anthra based & 114 & 1 & $0.239-1.908$ & 0.458 & 1 & $0.266-2.141$ & 0.596 \\
\hline $\mathrm{CT}+\mathrm{RT}$ & $\mathrm{CMF}$ & 21 & 0.675 & & & 0.596 & & \\
\hline \multirow[t]{2}{*}{$\mathrm{pN}$} & Negative & 95 & 1 & $1.441-5.436$ & 0.002 & 1 & $1.753-6.916$ & $<0.001$ \\
\hline & Positive & 39 & 2.798 & & & 3.482 & & \\
\hline \multirow[t]{3}{*}{$\mathrm{pCR}$} & Yes & 47 & 1 & $0.859-4.167$ & 0.113 & 1 & $0.897-4.764$ & 0.088 \\
\hline & No & 87 & 1.892 & & & 2.067 & & \\
\hline & $\mathrm{MVD} \leq 5.5$ & 47 & 1 & & & 1 & & \\
\hline \multirow[t]{2}{*}{ CD31 (n=92) } & MVD $>5.5$ & 45 & 1.073 & $0.4-2.5$ & 0.8 & 1.056 & $0.44-2.4$ & 0.9 \\
\hline & $\mathrm{MVD} \leq 100$ & 39 & 1 & & & 1 & & \\
\hline CD31 (n=52) & MVD $>100$ & 13 & 1.375 & $0.4-4.4$ & 0.59 & 1.38 & $0.4-4.5$ & 0.58 \\
\hline
\end{tabular}

were at least examined twice and all were positive for a control antibody, Ki-67. The representative images from a low-grade tumour and a high-grade tumour are shown (Figures 2 and 3). The disease free and overall survival for 92 patients is shown (Figure 4). The overall median MVD as determined by expression of CD31 was 5.5 and the mean MVD was 43.8. The median MVD of all 92 tumours was not significant as a prognostic factor probably due to sample size (data not shown). However, among the CD31 positive tumours $(n=52)$, the MVD (a)

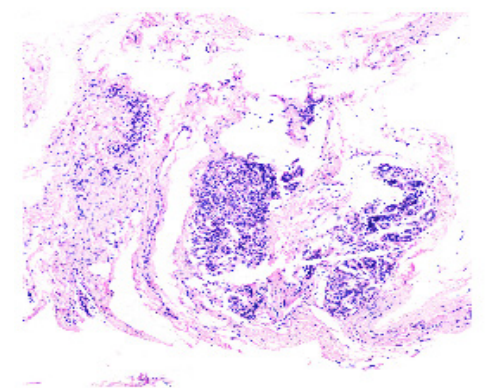

(c)

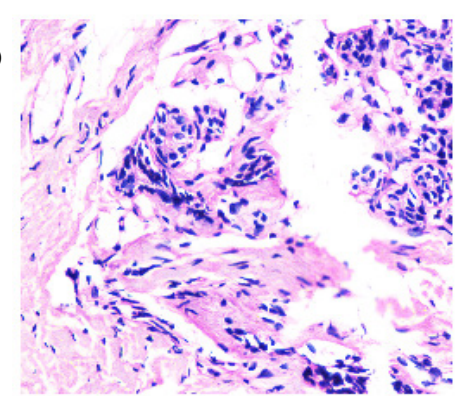

(b)

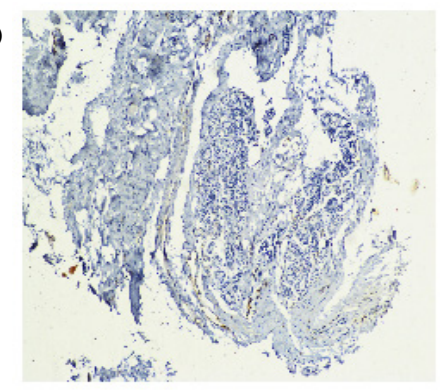

(d)

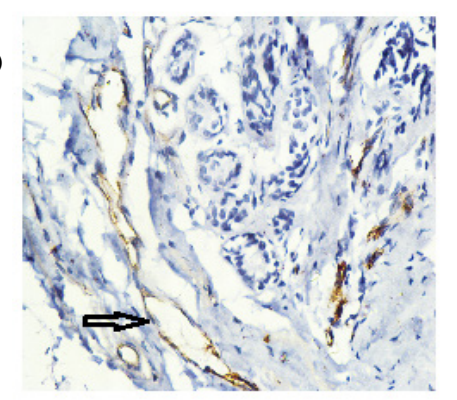

Figure 2. Expression of CD31 in a Low Grade LABC. H\&E stain (a\&c), CD31 (b\&d). The magnification is 10x (top panel) and 40x (bottom panel). 
(a)

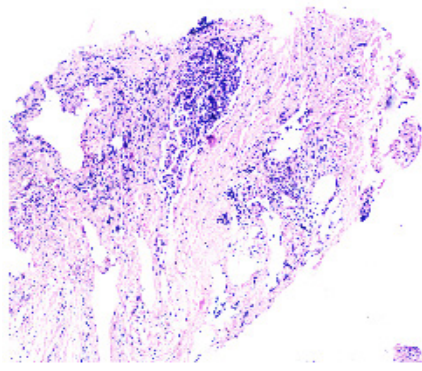

(c)

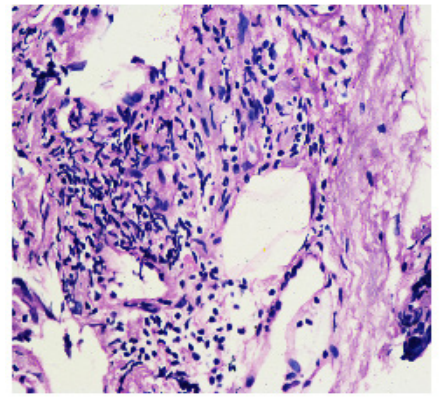

(b)

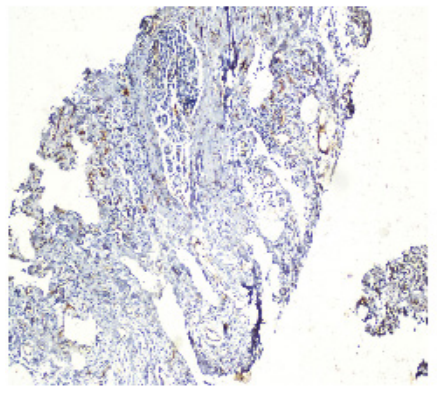

(d)

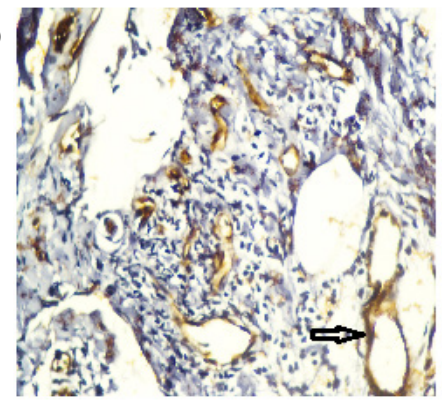

Figure 3. Expression of CD31 in a High Grade LABC. H\&E stain (a and c), CD31 (b and d). The magnification is 10x (top panel) and 40x (bottom panel)

ranged from 1 to 391 . Out of 52 tumours, 13 tumours had MVD greater than 100 and 39 tumours with MVD less than 100. The mean DFS and OS for the above 13 patients was lower (Figure 5) than that of the rest of the patients whose MVD of the tumours was less than 100. The univariate analysis by Cox regression model in this set of patients suggests that patients with tumours having MVD greater than 100 were at a high risk for recurrence than the rest of the patients (Table 2). However, it was not significant. Despite exhaustive examination for expression of CLEC14A in core needle biopsies, we were unable to identify CLEC14A+ endothelial cells. However, in a limited number of patients $(n=5)$ when we examined subsequent mastectomy specimens, there was unequivocal expression of CLEC14A if there was residual tumour (Figure 6).

\section{Discussion}

Locally advanced breast cancer forms a significant group of patients in any developing country including India. The proportion of LABC can be as high as 50-60\% in individual centres. Over the last 10 years, although breast cancer has become the most common in women at Chennai, the proportion of LABC has reduced. The management of these patients is quite challenging as initial

Table 3. Multivariate Analysis

\begin{tabular}{|c|c|c|c|c|c|c|}
\hline \multirow[t]{2}{*}{ Variable } & \multicolumn{3}{|c|}{ Model 1} & \multicolumn{3}{|c|}{ Model 2} \\
\hline & Hazard Ratio & $95 \% \mathrm{CI}$ & P Value & Hazard Ratio & $95 \% \mathrm{CI}$ & P Value \\
\hline \multicolumn{7}{|c|}{ A: Multivariate analysis for DFS } \\
\hline Age & 0.508 & $0.222-1.163$ & 0.109 & 0.518 & $0.226-1.186$ & 0.12 \\
\hline Clinical Tumor stage & 1.183 & $0.665-2.106$ & 0.568 & 0.961 & $0.529-1.746$ & 0.895 \\
\hline Clinical Node Stage & 2.445 & $1.246-4.798$ & 0.009 & & & \\
\hline $\mathrm{pN}$ & & & & 2.667 & $1.207-5.889$ & 0.015 \\
\hline pCR & 1.974 & $0.893-4.363$ & 0.093 & 1.119 & $0.439-2.850$ & 0.814 \\
\hline \multirow[t]{2}{*}{ Variable } & & Model 1 & & \multicolumn{3}{|c|}{ Model 2} \\
\hline & Hazard Ratio & $95 \% \mathrm{CI}$ & P Value & Hazard Ratio & $95 \% \mathrm{CI}$ & P Value \\
\hline \multicolumn{7}{|c|}{ B: Multivariate analysis for OS } \\
\hline Age & 0.287 & $0.101-0.819$ & 0.02 & 0.293 & $0.103-0.836$ & 0.022 \\
\hline Clinical stage & 1.687 & $0.920-3.090$ & 0.091 & 1.326 & $0.710-2.476$ & 0.377 \\
\hline Clinical Node Stage & 2.328 & $1.163-4.656$ & 0.017 & & & \\
\hline $\mathrm{pN}$ & & & & 3.155 & $1.335-7.452$ & 0.009 \\
\hline pCR & 2.145 & $0.928-4.960$ & 0.074 & 1.062 & $0.382-2.953$ & 0.908 \\
\hline
\end{tabular}




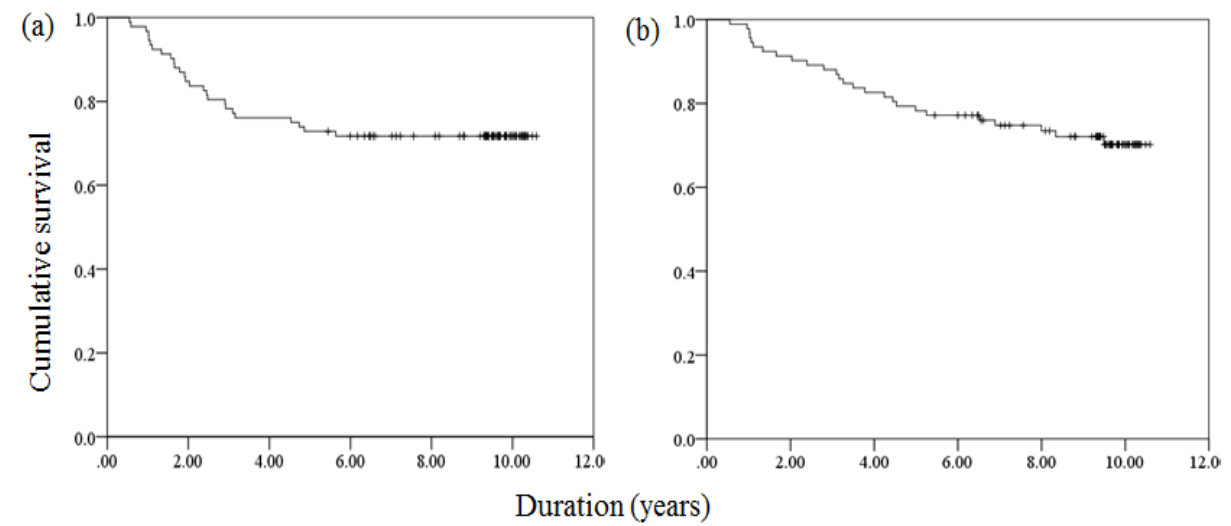

Figure 4. Survival Analysis of 92 Patients. (a) Disease-free and (b) Overall survival curves.

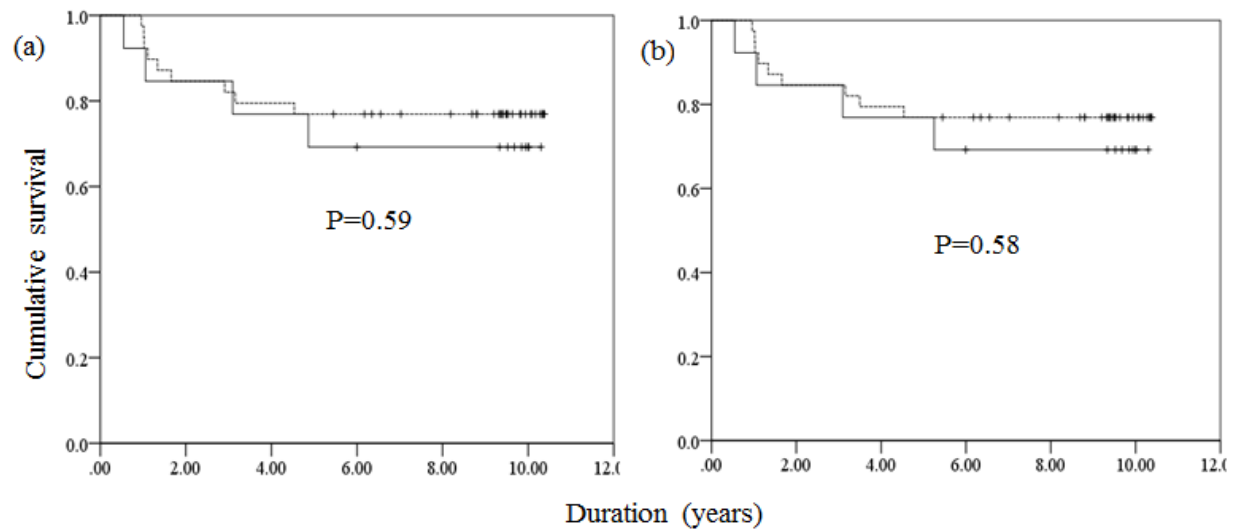

Figure 5. Correlation of MVD with Outcome. In patients whose initial biopsies showed expression of CD31 ( $\mathrm{n}=52$ ). The comparison is between patients whose tumours had MVD either greater than or less than 100. a) DFS and b) OS. Dotted line represents MVD $<100$ and straight line represents MVD $>100$.

(a)

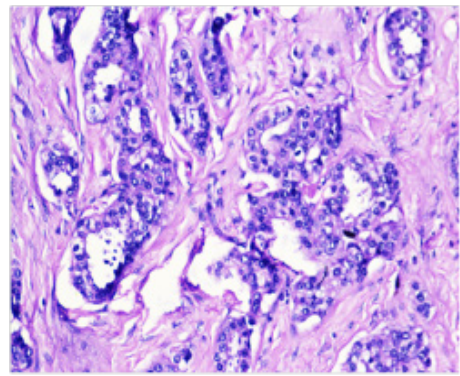

(c)

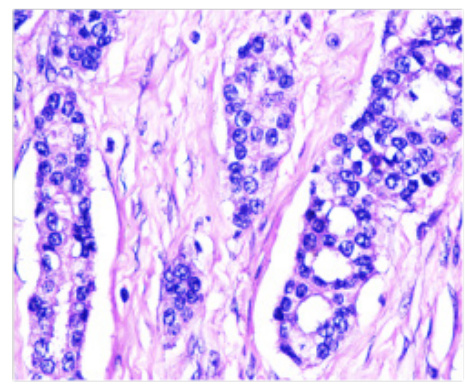

(b)

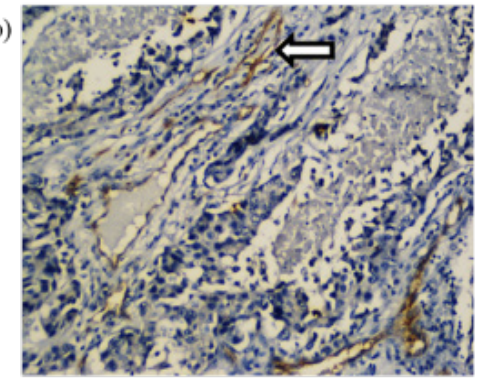

(d)

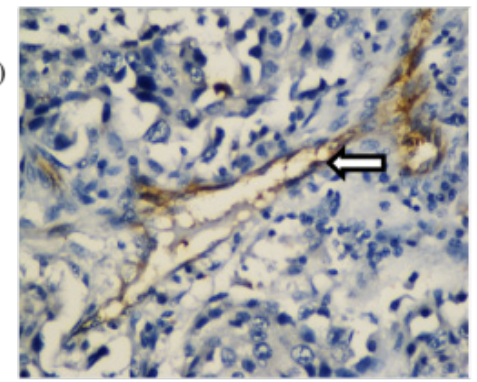

Figure 6. Expression of CLEC14A in a Mastectomy Specimen. H and E stain (a and c), CLEC14A (b and d). The magnification is 10x (top panel) and 40x (bottom panel).

surgery is not feasible. The treatment that has been evolved at Cancer Institute to treat patients with large LABC is to give concurrent chemo radiation. The long term outcomes with this approach are around $45 \%$ at 15 years (Shanta et al., 2008). The pathological complete response rates are higher when compared to patients receiving sequential chemotherapy followed by radiation. A randomised trial is in progress to assess both approaches.

Although, vascular density in tumours was shown to be an important prognostic factor in node negative breast 
cancer, subsequently this has been extended to all stages. However, most of these Clinicopathological studies were performed in patients with operable breast cancer. There is practically no report in the literature on MVD in LABC. The evaluation of MVD in LABC is challenging as the experiment has to be performed in a small core needle biopsy as opposed to a mastectomy specimen. It may also not be representative of the whole tumour. However, performing IHC after mastectomy is confounded by effects of treatment.

We found that in univariate analysis, the prognostic factors such as age, clinical node stage, and pathological nodal status significantly correlated with disease free and overall survival. We also found that the patients $(n=52)$, whose tumours had very high MVD (greater than 100), had poor progression free and overall survival when compared to the rest of the patients, although it was not significant. There are previous reports which showed vascular counts by CD31 correlated with relapse free survival in primary operable early stage breast cancers (Horak et al., 1992; Toi et al., 1993). A meta-analysis of 87 studies demonstrated that MVD was a significant prognostic factor in patients with breast cancer. This study also reported that MVD as assessed by CD31 or CD34 might be a better prognostic factor than Factor VIII (Uzzan et al., 2004). A more recent study showed that increased MVD of CD31 in whole sections of breast biopsies was significantly associated $(\mathrm{OR}=1.69,95 \% \mathrm{CI}=1.17-2.44)$ with in situ or invasive breast cancer (Felix et al., 2016). However, in our study, MVD as measured by expression of CD31 was not a significant prognostic factor for overall survival in the 92 patients. Similar results were also demonstrated in other studies that MVD of CD31 did not correlate with clinical outcome (Honkoop et al., 1998; Mayers et al., 1998; Charpin et al., 2004). MVD determined by CD105 was found to predict disease free survival in patients with stage I and II diseases whereas only hormone receptors were found to correlate with overall survival in patients with stage III disease (Rau et al., 2012). However,vascular invasion was reported to be a significant prognostic factor in basal and triple negative breast cancer by univariate and multivariate analyses (Mohammed et al., 2011). Hence, the previous reports on MVD in breast tumours are not consistent. It is important to note that more careful appraisal of mean vascular density and its relation with different subtypes have to be performed. This may identify subgroups where vascular density correlates with outcome.

Our next objective was to study if the MVD as determined by the newly reported endothelial marker, C-type lectin 14A (CLEC14A) correlates with survival in LABC. We have evaluated MVD as detected by expression of CLEC14A in ovarian tumours and found that it was significantly lower than that of CD31 and CD105 (Krishna Priya et al., 2017). Surprisingly, there was no expression of this marker in core needle biopsies from LABC and there was positive staining only in mastectomy specimens. It was reported that CLEC14A could be a more specific marker for tumour blood vessels (Mura et al., 2012). The lack of expression of CLEC14A could be due to two reasons. Firstly, the size of the tissue from biopsies was not representative of the whole tumour and the second reason could be that CLEC14A is expressed at the later stages of tumour progression.

Although there are conflicting reports on the prognostic significance of MVD in breast cancer, antiangiogenic therapy is being used in treating patients with this tumour type. The phase 3 trial from 10 countries, with bevacizumab with capecitabine and bevacizumab alone, concluded that the former benefits both progressionfree survival and overall survival in patients with Her2 negative tumours (Gligorov et al., 2014). However, other trials such as (E2100, AVADO, RIBBON-1 and RIBBON-2) showed that this therapy does not improve overall survival of patients with breast cancer (Seddon et al., 2014). Despite this, there may be a benefit with bevacizumab, when the initial MVD is higher since it enhanced vascular normalization. Therefore, MVD before treatment could be a predictive marker in order to assess the response to bevacizumab in breast cancer and new therapies should be identified to normalize vessels (Tolaney et al., 2015). Overall, the inability to show that MVD is a significant prognostic factor is probably related to the number of tumours. However it is equally possible, that as angiogenesis is not uniform within a tumour, and the biopsies may not be representative of active site of angiogenesis. This report sets the background for a more detailed evaluation of additional markers to evaluate MVD in LABC.

\section{Funding statement}

The study was supported by Department of Biotechnology and Ms.S.Krishna Priya was a recipient of Senior Research Fellowship from Indian Council of Medical Research, Government of India.

\section{Acknowledgements}

We acknowledge the help and support of the staff of pathology and tumour registry at Cancer Institute (WIA), Chennai for collecting the clinical data and acquisition of tumour blocks and preparation of slides.

\section{References}

Bouck N, Stellmach V, Hsu SC (1996). How tumors become angiogenic. Adv Cancer Res, 69, 135-74.

Charpin C, Dales JP, Garcia S, et al (2004). Tumor neoangiogenesis by CD31 and CD105 expression evaluation in breast carcinoma tissue microarrays. Clin Cancer Res, 10, 5815-9.

Cox DR, Oakes D (1984). Analysis of Survival Data, Chapman and Hall, London.

Dachs GU, Chaplin DJ (1998). Microenvironmental control of gene expression: implications for tumor angiogenesis, progression, and metastasis. Semin Radiat Oncol, 8, 208-16.

El-Gohary YM, Silverman JF, Olson PR, et al (2007). Endoglin (CD105) and vascular endothelial growth factor as prognostic markers in prostatic adenocarcinoma. Am J Clin Pathol, 127, 572-9.

Felix AS, Lenz P, Pfeiffer RM, et al (2016). Relationships between mammographic density, tissue microvessel density, and breast biopsy diagnosis. Breast Cancer Res, 18, 88.

Ferlay J, Shin HR, Bray F, et al (2010). Estimates of worldwide burden of cancer in 2008: GLOBOCAN 2008. Int J Cancer, 
127, 2893-917.

Folkman J (1971). Tumor angiogenesis: therapeutic implications. N Engl J Med, 285, 1182-6.

Fox SB, Harris AL (2004). Histological quantitation of tumour angiogenesis. APMIS, 112, 413-30.

Gligorov J, Doval D, Bines J, et al (2014). Maintenance capecitabine and bevacizumab versus bevacizumab alone after initial first-line bevacizumab and docetaxel for patients with HER2-negative metastatic breast cancer (IMELDA): a randomised, open-label, phase 3 trial. Lancet Oncol, 15, 1351-60.

Hanahan D, Folkman J (1996). Patterns and emerging mechanisms of the angiogenic switch during tumorigenesis. Cell, 86, 353-64.

Heath VL, Bicknell R (2009). Anticancer strategies involving the vasculature. Nat Rev Clin Oncol, 6, 395-404.

Honkoop AH, van Diest PJ, de Jong JS, et al (1998). Prognostic role of clinical, pathological and biological characteristics in patients with locally advanced breast cancer. Br J Cancer, 77, 621-6.

Horak ER, Leek R, Klenk N, et al (1992). Angiogenesis, assessed by platelet/endothelial cell adhesion molecule antibodies, as indicator of node metastases and survival in breast cancer. Lancet, 340, 1120-4.

Kaplan EL, Meier P (1958). Nonparametric Estimation from Incomplete Observations. $J$ Am Stat Assoc, 53, 457-81.

Krishna Priya S, Kumar K, Hiran KR, et al (2017). Expression of a novel endothelial marker, C-type lectin 14A, in epithelial ovarian cancer and its prognostic significance. Int J Clin Oncol, 22, 107-17.

Mayers MM, Seshadri R, Raymond W, et al (1998). Tumor microvascularity has no independent prognostic significance for breast cancer. Pathology, 30, 105-10.

Mohammed RA, Ellis IO, Mahmmod AM, et al (2011). Lymphatic and blood vessels in basal and triple-negative breast cancers: characteristics and prognostic significance. Mod Pathol, 24, 774-85.

Mura M, Swain RK, Zhuang X, et al (2012). Identification and angiogenic role of the novel tumor endothelial marker CLEC14A. Oncogene, 31, 293-305.

Rau KM, Huang CC, Chiu TJ, et al (2012). Neovascularization evaluated by CD105 correlates well with prognostic factors in breast cancers. Exp Ther Med, 4, 231-6.

Seddon AN, Cuellar S, Haaf CM (2014). The life, death, and attempted rebirth of bevacizumab in breast cancer. $J$ Oncol Pharm Pract, 20, 433-44.

Shanta V, Swaminathan R, Rama R, et al (2008). Retrospective analysis of locally advanced noninflammatory breast cancer from Chennai, South India, 1990-1999. Int J Radiat Oncol Biol Phys, 70, 51-8.

Shetty P (2012). India faces growing breast cancer epidemic. Lancet, 379, 992-3.

Siemann DW (2011). The unique characteristics of tumor vasculature and preclinical evidence for its selective disruption by Tumor-Vascular Disrupting Agents. Cancer Treat Rev, 37, 63-74.

Toi M, Kashitani J, Tominaga T (1993). Tumor angiogenesis is an independent prognostic indicator in primary breast carcinoma. Int $J$ Cancer, 55, 371-4.

Tolaney SM, Boucher Y, Duda DG, et al (2015). Role of vascular density and normalization in response to neoadjuvant bevacizumab and chemotherapy in breast cancer patients. Proc Natl Acad Sci U S A, 112, 14325-30.

Uzzan B, Nicolas P, Cucherat M, et al (2004). Microvessel density as a prognostic factor in women with breast cancer: a systematic review of the literature and meta-analysis. Cancer Res, 64, 2941-55.
Weidner N (2000). Angiogenesis as a predictor of clinical outcome in cancer patients. Hum Pathol, 31, 403-5.

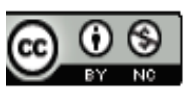

This work is licensed under a Creative Commons AttributionNon Commercial 4.0 International License. 